\title{
Flexible NiO-Graphene-Carbon Fiber Mats Containing Multifunctional Graphene for High Stability and High Specific Capacity Lithium-Ion Storage
}

Zhongqi Wang, ${ }^{1}$ Ming Zhang, ${ }^{2 *}{ }^{\text {Ji }}$ Zhou $^{1 *}$

${ }^{1}$ State Key Lab of New Ceramics and Fine Processing, Department of Materials Science and Engineering, Tsinghua University, Beijing 100084, China. Email: zhouji@mail.tsinghua.edu.cn

${ }^{2}$ Key Laboratory for Micro-Nano Optoelectronic Devices of Ministry of Education, School of Physics and Electronics, State Key Laboratory for Chemo/Biosensing and Chemometrics, Hunan University, Changsha 410082, China. Email: zhangming@hnu.edu.cn 


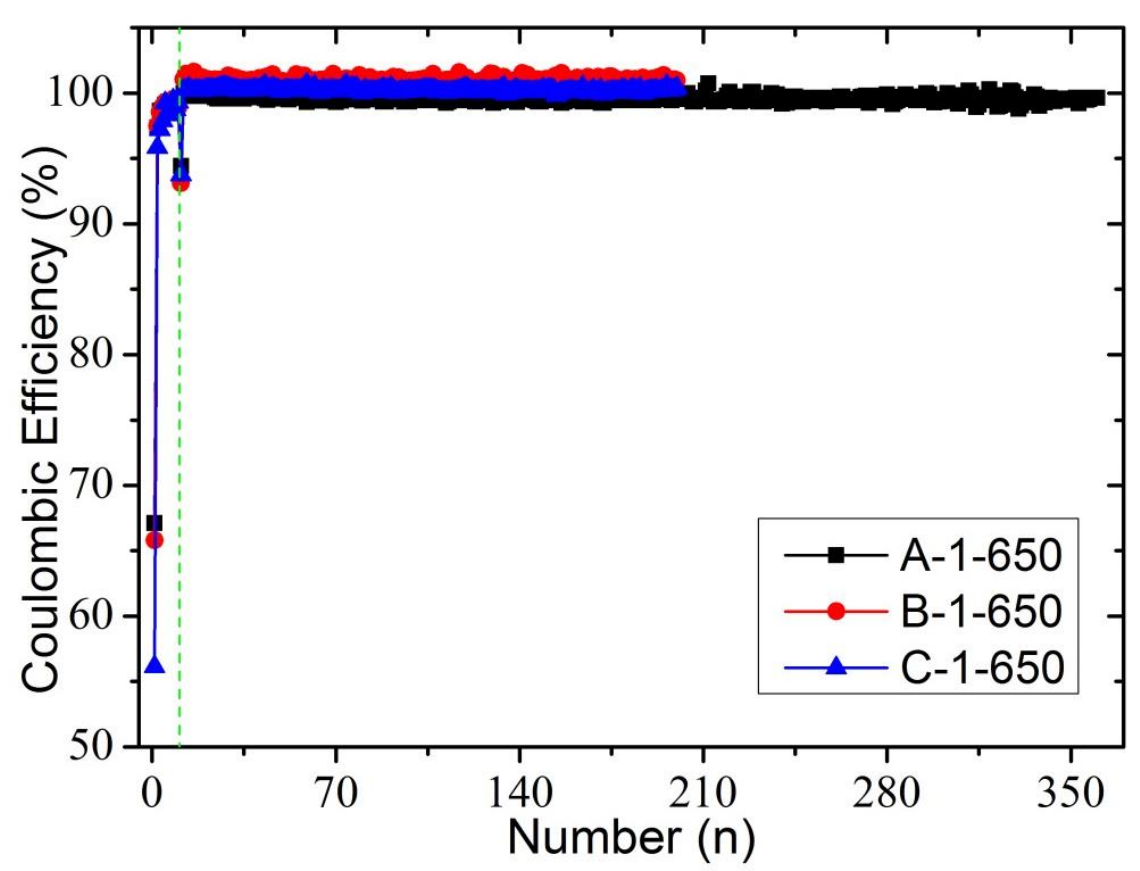

Figure S1. Coulombic efficiency of NiO-graphene-carbon fiber mats with different concentration of $\mathrm{Ni}$ obtained at $650{ }^{\circ} \mathrm{C}$.
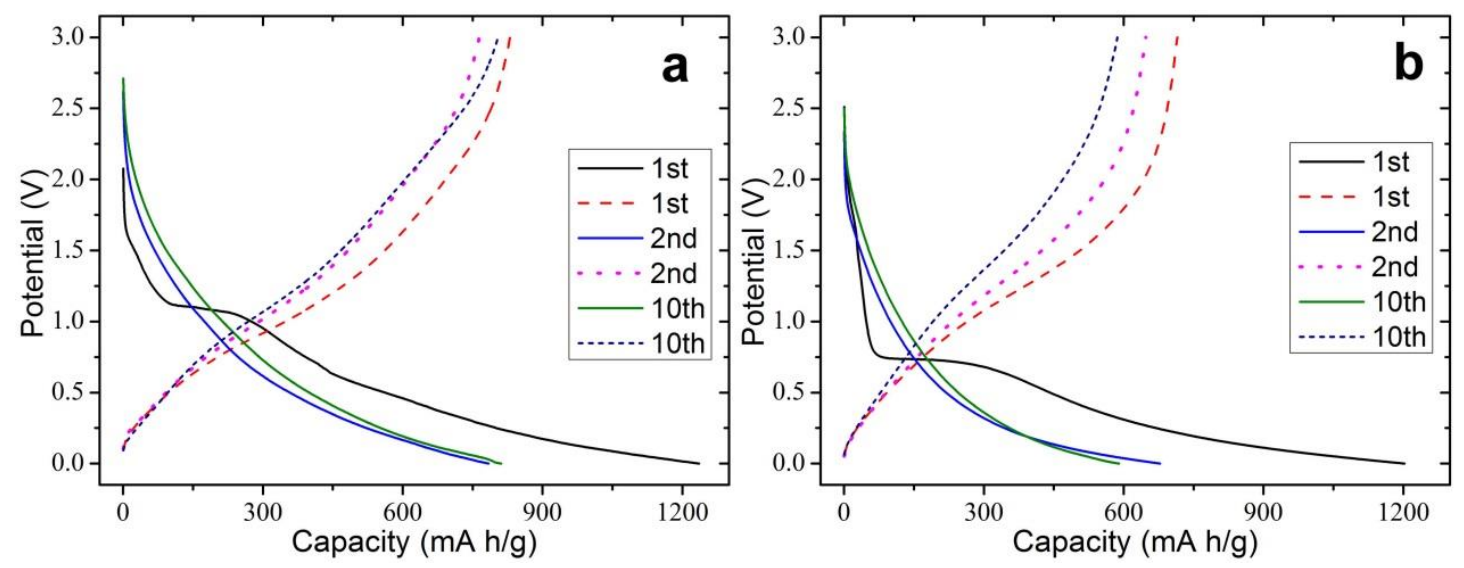

Figure S2. Typical charge-discharge curves of (a) A-1-650 and (b) F-650 at the $1^{\text {st }}, 2^{\text {nd }}$, and $10^{\text {th }}$ cycles. 


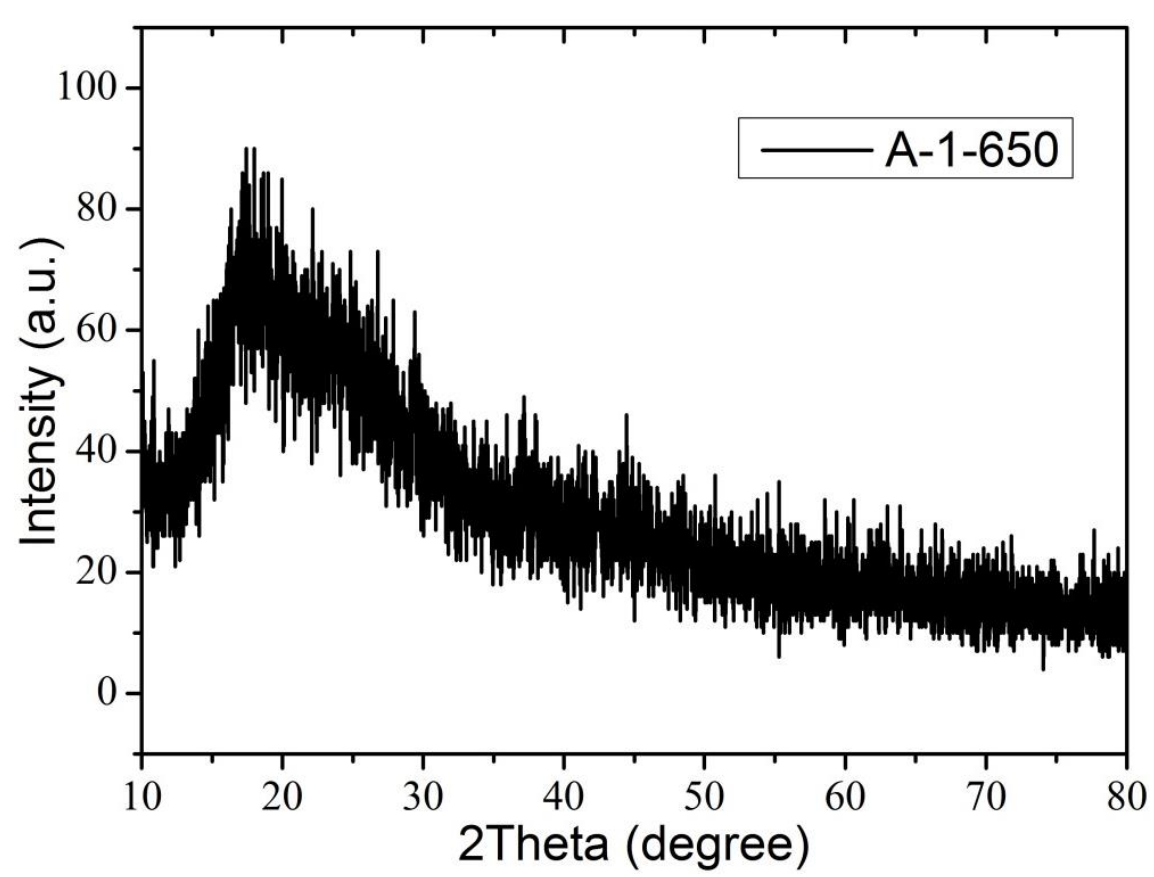

Figure S3. XRD patterns of NGCs (A-1-650). No obvious peak can be indexed to $\mathrm{NiO}$.

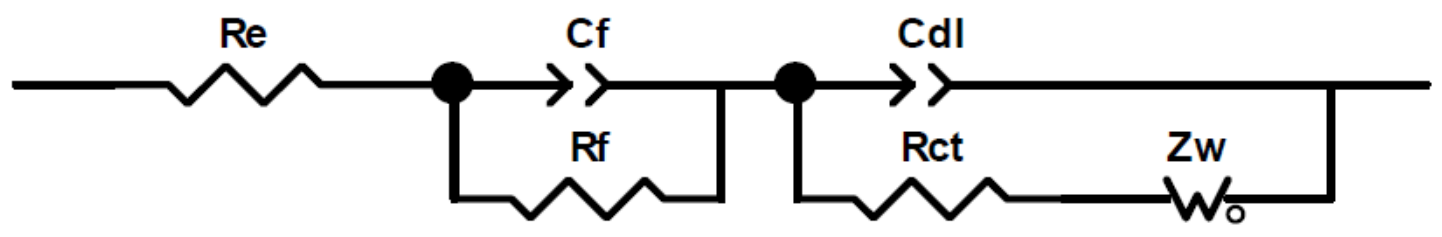

Figure S4. The equivalent circuit to fit the Nyquist plots. 


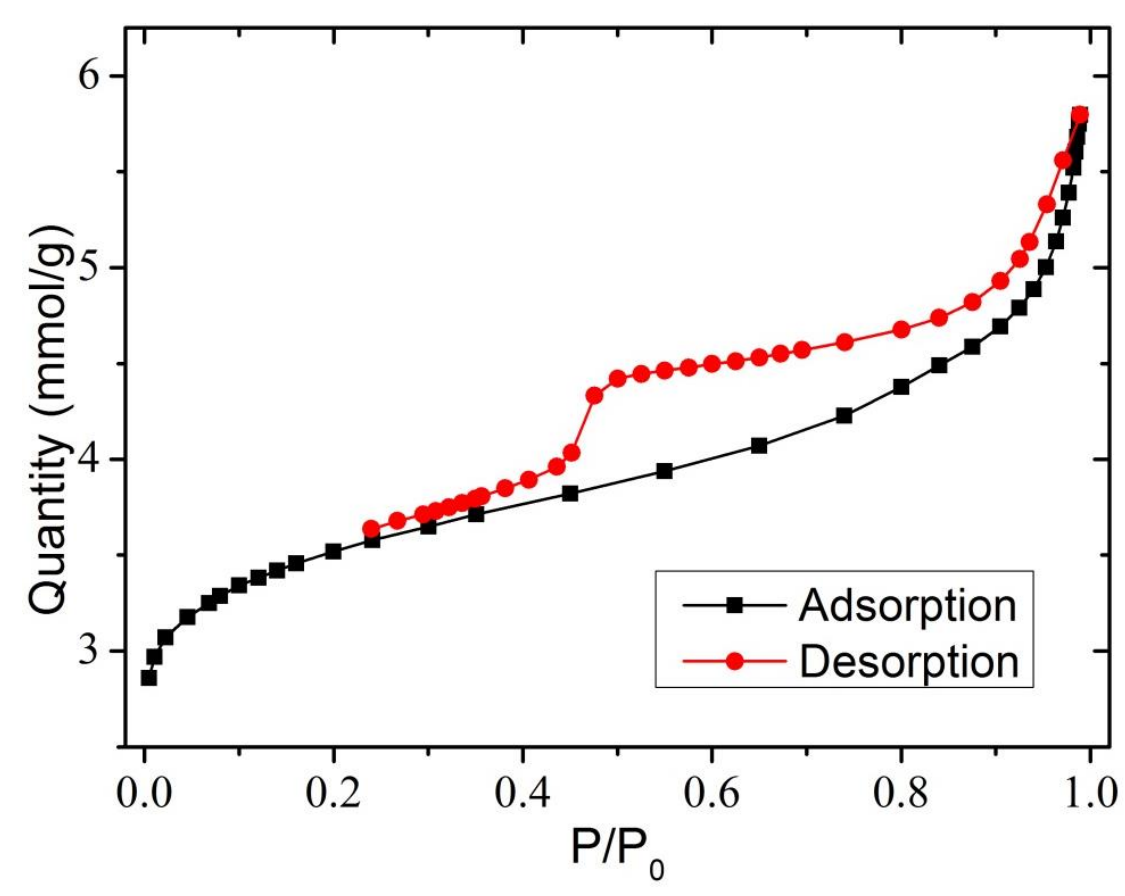

Figure S5. The nitrogen adsorption-desorption isotherms of the carbon fibers arising from A-1-650 after removing $\mathrm{NiO}$.

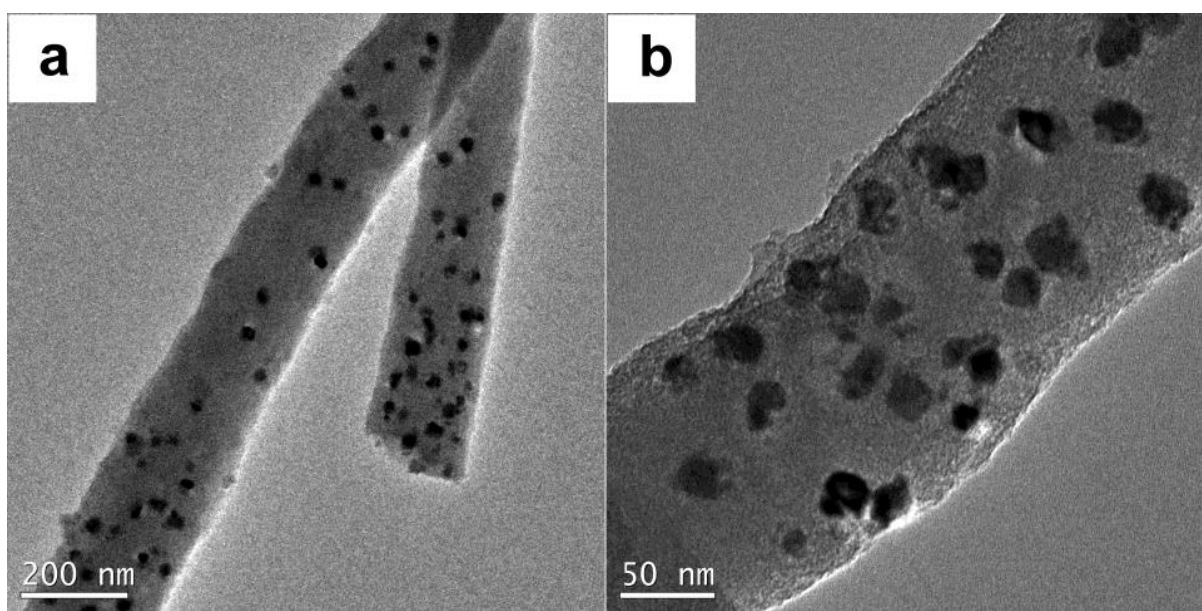

Figure S6. (a) and (b) show the TEM images of NGCs (A-1-650) after cyclic tests. It can be found that the smooth fibers are integral. The size of nanoparticles inside the carbon nanofibers are approximate $20-30 \mathrm{~nm}$, indicating no obvious changes during lithiation/delithiation cycles. So the A-1-650 NGCs show good cyclic stability for the storage of $\mathrm{Li}^{+}$. 
According to the I-V curves, the electrical conductivity could be calculated by the following equation:

$$
\sigma=\frac{l \cdot I}{S \cdot U}
$$

Where $\sigma$ is the electrical conductivity, $l$ is the length of mats, $S$ is the cross sectional area, $I$ is the current and $U$ is the voltage.

Table S1. Conductivities of NGCs (A-1-650, A-2-650) and $\mathrm{NiO}_{\mathrm{x}}-\mathrm{C}$ (A-2-650).

\begin{tabular}{ll}
\hline Samples & Conductivity $(\mathrm{S} / \mathrm{m})$ \\
\hline A-1-600 & $1.5 \times 10^{-5}$ \\
A-1-650 & $1.2 \times 10^{-3}$ \\
A-2-650 & $1.6 \times 10^{-3}$ \\
\hline
\end{tabular}

